\title{
Nilpotent $\mathcal{N}=1$ tensor multiplet
}

\section{Sergei M. Kuzenko}

Department of Physics M013, The University of Western Australia, 35 Stirling Highway, Crawley W.A. 6009, Australia

E-mail: sergei.kuzenko@uwa.edu.au

ABSTRACT: We propose a nilpotent $\mathcal{N}=1$ tensor multiplet describing two fields, which are the Goldstino and the axion, the latter being realised in terms of the field strength of a gauge two-form. This supersymmetric multiplet is formulated in terms of a deformed real linear superfield, subject to a cubic nilpotency condition. Its couplings to a super YangMills multiplet and supergravity are presented. To define a nilpotent tensor multiplet in the locally supersymmetric case, one has to make use of either real or complex three-form supergravity theories, which are variant realisations of the old minimal formulation for $\mathcal{N}=1$ supergravity.

Keywords: Supergravity Models, Superspaces, Supersymmetry and Duality, Supersymmetry Breaking

ARXIV EPrint: 1712.09258 


\section{Contents}

1 Introduction 1

2 A generalisation of the linear-chiral duality 4

3 New nilpotent multiplet $\quad 5$

4 Nilpotent tensor multiplet coupled to a three-form multiplet $\quad 6$

$\begin{array}{llr}5 & \text { Coupling to three-form supergravity } & 8\end{array}$

$\begin{array}{ll}\text { A More duality transformations } & 10\end{array}$

\section{Introduction}

Following the construction of inflationary models with nilpotent superfields [1-4], in the last two years there has been much interest in models for de Sitter supergravity, see [5-14] and references therein. ${ }^{1}$ They are off-shell models for spontaneously broken local supersymmetry obtained by coupling $\mathcal{N}=1$ supergravity to various nilpotent Goldstino superfields. One of the reasons for the interest in such theories is that a positive contribution to the cosmological constant is generated once the local supersymmetry becomes spontaneously broken. For instance, if the supergravity multiplet is coupled to an irreducible Goldstino superfield $^{2}[7,12,15,19,20]$ (with the Volkov-Akulov Goldstino [21-23] being the only independent component field of the superfield), a universal positive contribution to the cosmological constant is generated, which is proportional to $f^{2}$, with the parameter $f$ setting the scale of supersymmetry breaking. The same positive contribution is generated by the reducible Goldstino superfields used in the models studied in $[5,6,13]$. There is one special reducible Goldstino superfield, the nilpotent three-form multiplet introduced in $[11,14]$, which yields a dynamical contribution to the cosmological constant.

Historically, the first off-shell model for de Sitter supergravity was constructed by Lindström and Roček in 1979 [15]. They made use of the irreducible nilpotent chiral Goldstino superfield proposed by Roček [16]. As shown in [12, 13], on the mass shell this model is equivalent to the one advocated in $[5,6]$, which made use of the reducible nilpotent chiral Goldstino superfield proposed in [17, 18].

Since we live in a universe dominated by dark energy and dark matter, and since dark energy can be sourced by a small positive cosmological constant, it is remarkable that spontaneously broken local supersymmetry provides a mechanism to generate a universal

\footnotetext{
${ }^{1}$ The terminology "de Sitter supergravity" was coined in [5].

${ }^{2}$ The notion of irreducible and reducible Goldstino superfields was introduced in [12].
} 
positive contribution to the cosmological constant, which is associated with the Goldstino. As concerns the known candidates for dark matter (see, e.g., [24] for a review), the axion field is among the most interesting ones. It is natural to wonder whether there exists a constrained supermultiplet containing both the Goldstino and the axion. Such a supermultiplet is proposed in this paper.

In the case of $\mathcal{N}=1$ supersymmetry, every known scalar Goldstino superfield $X$, irreducible or reducible, obeys the quadratic nilpotency condition

$$
X^{2}=0
$$

These Goldstino superfields include: (i) the irreducible chiral scalar proposed in [16, 25]; (ii) the reducible chiral scalar of $[17,18]$; (iii) the deformed complex linear scalar introduced in [20]; (iv) the complex linear scalar of [26-28]; (v) the irreducible real scalar proposed in [12]; (vi) the reducible real scalar of [13]. In the (v) and (vi) cases, there are actually three nilpotency conditions [12, 13]:

$$
\begin{aligned}
V^{2} & =0, \\
V D_{A} D_{B} V & =0, \\
V D_{A} D_{B} D_{C} V & =0,
\end{aligned}
$$

where $D_{A}=\left(\partial_{a}, D_{\alpha}, \bar{D}^{\dot{\alpha}}\right)$ are the covariant derivatives of Minkowski superspace $\mathbb{M}^{4 \mid 4}{ }^{3}$

The standard linear multiplet [29], which is described by a real scalar superfield $G=\bar{G}$ constrained by $\bar{D}^{2} G=0$, cannot be subject to any nilpotency condition in order to describe a Goldstino superfield. The point is that the $\mathcal{N}=1$ tensor multiplet [30], for which $G$ originates as the gauge invariant field strength, has no auxiliary field. Therefore, the constraint $\bar{D}^{2} G=0$ has to be deformed if we wish to use a linear-type superfield to embed the Goldstino into. In order to get a feeling for a suitable deformation, let us consider the simplest model for spontaneously broken supersymmetry, realised in terms of a chiral scalar $\Phi$ and its conjugate $\bar{\Phi}$, with action

$$
S_{\mathrm{PM}}=\int \mathrm{d}^{4} x \mathrm{~d}^{2} \theta \mathrm{d}^{2} \bar{\theta} \bar{\Phi} \Phi-\left\{f \int \mathrm{d}^{4} x \mathrm{~d}^{2} \theta \Phi+\text { c.c. }\right\}, \quad \bar{D}_{\dot{\alpha}} \Phi=0,
$$

where $f$ is a non-zero parameter of mass dimension +2 . This theory possesses a dual formulation described in [20]. Specifically, associated with (1.3) is the first-order model

$$
\begin{aligned}
S_{\text {first-order }}^{(\Sigma)} & =\int \mathrm{d}^{4} x \mathrm{~d}^{2} \theta \mathrm{d}^{2} \bar{\theta}(\bar{U} U-\Sigma U-\bar{\Sigma} \bar{U}), \\
-\frac{1}{4} \bar{D}^{2} \Sigma & =f
\end{aligned}
$$

in which $U$ is a complex unconstrained superfield, and $\Sigma$ is a deformed complex linear superfield constrained by (1.4b). Varying (1.4a) with respect to $\Sigma$ gives $U=\Phi$, and then the action (1.4a) reduces to (1.3). Therefore, the supersymmetric theories (1.3) and (1.4)

\footnotetext{
${ }^{3}$ A real scalar Goldstino superfield was briefly discussed in ref. [15] and later reviewed in [19]. However, only the constraint $(1.2 \mathrm{a})$ was explicitly given in these publications.
} 
are equivalent. On the other hand, the auxiliary superfields $U$ and $\bar{U}$ can be integrated out from the action (1.4a) resulting with

$$
S_{\Sigma}=-\int \mathrm{d}^{4} x \mathrm{~d}^{2} \theta \mathrm{d}^{2} \bar{\theta} \bar{\Sigma} \Sigma
$$

The Goldstino superfield model of [20] made use of $\Sigma$ subject to the holomorphic constraints

$$
\Sigma^{2}=0, \quad-\frac{1}{4} \Sigma \bar{D}^{2} D_{\alpha} \Sigma=f D_{\alpha} \Sigma .
$$

There exists a different dual formulation for (1.3). Let us consider the following firstorder action

$$
\begin{aligned}
S_{\text {first-order }}^{(\mathfrak{G})} & =\int \mathrm{d}^{4} x \mathrm{~d}^{2} \theta \mathrm{d}^{2} \bar{\theta}\left(\frac{1}{2} V^{2}-\mathfrak{G} V\right), \\
-\frac{1}{4} \bar{D}^{2} \mathfrak{G} & =f, \quad \overline{\mathfrak{G}}=\mathfrak{G} .
\end{aligned}
$$

Here $V$ is a real unconstrained superfield, and $\mathfrak{G}$ is a deformed real linear superfield constrained by (1.7b). The supersymmetric theories (1.3) and (1.7) are equivalent. Indeed, varying $S_{\text {first-order }}^{(\mathfrak{G})}$ with respect to $\mathfrak{G}$ gives $V=\Phi+\bar{\Phi}$, and then the action (1.7a) reduces to (1.3). On the other hand, we can integrate out the auxiliary superfield $V$ from $S_{\text {first-order }}^{(\mathfrak{G})}$ to end up with

$$
S_{\mathfrak{G}}=-\frac{1}{2} \int \mathrm{d}^{4} x \mathrm{~d}^{2} \theta \mathrm{d}^{2} \bar{\theta} \mathfrak{G}^{2}
$$

It is easy to see that requiring the deformed real linear superfield $\mathfrak{G}$ to obey the quadratic nilpotency condition (1.1) does not allow us to eliminate the scalar field, $\left.\mathfrak{G}\right|_{\theta=0}$, contained in $\mathfrak{G}$. However, this becomes possible if we subject $\mathfrak{G}$ to the cubic nilpotency condition

$$
\mathfrak{G}^{3}=0
$$

The resulting supermultiplet contains only two fields, which are the Goldstino and the axion, the latter being described in terms of a gauge two-form. In this paper we will study the properties of this supermultiplet and its generalisations, including its couplings to Yang-Mills supermultiplets and supergravity.

It should be pointed out that cubic nilpotency conditions have been discussed in the literature $[18,31-34]$ for two $\mathcal{N}=1$ superfields, one of which is the nilpotent chiral scalar $X$ subject to the only constraint $(1.1)$, as proposed in $[17,18]$. Cubic nilpotency conditions for a single $\mathcal{N}=2$ Goldstino superfield have been proposed in [13, 35, 36].

This paper is organised as follows. In section 2 we show how deformed real linear superfields $(1.7 \mathrm{~b})$ originate within a framework generalising the linear-chiral duality. Our new nilpotent multiplet is described in section 3. Its couplings to a three-form multiplet, a super Yang-Mills multiplet and three-form supergravity are presented in sections 4 and 5 . Finally, the appendix is devoted to some generalisations of the duality transformations described above. 


\section{A generalisation of the linear-chiral duality}

We start by recalling the linear-chiral duality as described in [37]. Consider a general two-derivative model for a self-interacting $\mathcal{N}=1$ tensor multiplet [30]

$$
S[G]=\int \mathrm{d}^{4} x \mathrm{~d}^{2} \theta \mathrm{d}^{2} \bar{\theta} \mathcal{F}(G), \quad D^{2} G=\bar{D}^{2} G=0,
$$

where $G=\bar{G}$ is the gauge-invariant field strength of the tensor multiplet, and $\mathcal{F}(x)$ is a smooth function of a real variable $x .{ }^{4}$ The choice $\mathcal{F}(x)=-x^{2}$ corresponds to the free tensor multiplet [30], while another choice $\mathcal{F}(x)=-x \ln x$ corresponds to the so-called improved tensor multiplet [38].

We associate with (2.1) the following first-order model

$$
S[K, \Phi, \bar{\Phi}]=\int \mathrm{d}^{4} x \mathrm{~d}^{2} \theta \mathrm{d}^{2} \bar{\theta}\{\mathcal{F}(K)-(\Phi+\bar{\Phi}) K\}, \quad \bar{D}_{\dot{\alpha}} \Phi=0 .
$$

Here the dynamical variables are a real unconstrained superfield $K$, a chiral scalar $\Phi$ and its complex conjugate $\bar{\Phi}$. Varying $S[K, \Phi, \bar{\Phi}]$ with respect to the Lagrange multiplier $\Phi$ gives the equation of motion $\bar{D}^{2} K=0$, and hence $K=G$. Then the second term in the integrand (2.2) drops out, and we are back to the tensor multiplet model (2.1). Therefore, the theories (2.1) and (2.2) are equivalent. On the other hand, we can vary (2.2) with respect to $K$ resulting in the equation of motion

$$
\mathcal{F}^{\prime}(K)=\Phi+\bar{\Phi}
$$

Assuming that $\mathcal{F}(x)$ possesses a Legendre transform, this equation allows us to express $K$ as a function of $\Phi$ and $\bar{\Phi}$, and then (2.2) turns into the dual action

$$
S_{\mathrm{D}}[\Phi, \bar{\Phi}]=\int \mathrm{d}^{4} x \mathrm{~d}^{2} \theta \mathrm{d}^{2} \bar{\theta} \mathcal{F}_{\mathrm{D}}(\Phi+\bar{\Phi}),
$$

where $\mathcal{F}_{\mathrm{D}}$ is the Legendre transform of $\mathcal{F}$. This supersymmetric nonlinear $\sigma$-model is a dual formulation for the tensor multiplet theory (2.1).

There exists a variant realisation of the scalar multiplet known as the three-form multiplet [39]. It is obtained by replacing the chiral scalar $\Phi$ with $\chi$ given by

$$
\chi=-\frac{1}{4} \bar{D}^{2} U, \quad \bar{U}=U .
$$

Now, starting from the nonlinear $\sigma$-model (2.4), we may construct a theory of selfinteracting three-form multiplet

$$
S_{\alpha}[\chi, \bar{\chi}]=\int \mathrm{d}^{4} x \mathrm{~d}^{2} \theta \mathrm{d}^{2} \bar{\theta} \mathcal{F}_{\mathrm{D}}\left(\mathrm{e}^{\mathrm{i} \alpha} \chi+\mathrm{e}^{-\mathrm{i} \alpha} \bar{\chi}\right)
$$

for some parameter $\alpha \in \mathbb{R}$. Since the prepotential $U$ in (2.5) is real, we cannot absorb the phase factor $\mathrm{e}^{\mathrm{i} \alpha}$ into $\chi$, unlike the case of $\Phi$. Let us make the same replacement, $\Phi \rightarrow \mathrm{e}^{\mathrm{i} \alpha} \chi$, in the first-order action (2.2), resulting with ${ }^{5}$

$$
S_{\alpha}[K, \chi, \bar{\chi}]=\int \mathrm{d}^{4} x \mathrm{~d}^{2} \theta \mathrm{d}^{2} \bar{\theta}\left\{\mathcal{F}(K)-\left(\mathrm{e}^{\mathrm{i} \alpha} \chi+\mathrm{e}^{-\mathrm{i} \alpha} \bar{\chi}\right) K\right\} .
$$

\footnotetext{
${ }^{4}$ Following [29], $G$ is called a real linear superfield.

${ }^{5}$ In the case $\mathcal{F}(K) \propto K^{2}$, the first-order action (2.7) was considered in [40].
} 
This theory is equivalent to (2.6). However, varying the action (2.7) with respect to $U$ gives the equation

$$
\mathrm{e}^{\mathrm{i} \alpha} \bar{D}^{2} K+\mathrm{e}^{-\mathrm{i} \alpha} D^{2} K=0
$$

which is equivalent to

$$
\bar{D}^{2} K=\mathrm{ie}^{-\mathrm{i} \alpha} m, \quad m=\bar{m}=\text { const },
$$

for some real parameter $m$. This equation defines a deformed real linear multiplet.

\section{New nilpotent multiplet}

We consider a real scalar superfield $\boldsymbol{G}=\overline{\boldsymbol{G}}$ subject to a deformed linear constraint

$$
-\frac{1}{4} D^{2} \boldsymbol{G}=\bar{\mu}=\mathrm{const} \quad \Longleftrightarrow \quad-\frac{1}{4} \bar{D}^{2} \boldsymbol{G}=\mu=\mathrm{const},
$$

for some non-zero complex parameter $\mu$. The general solution to this constraint is

$$
\boldsymbol{G}=\varphi+\theta^{\alpha} \psi_{\alpha}+\bar{\theta}_{\dot{\alpha}} \bar{\psi}^{\dot{\alpha}}+\theta^{2} \bar{\mu}+\bar{\theta}^{2} \mu+\theta \sigma^{a} \bar{\theta} H_{a}+\frac{\mathrm{i}}{2} \theta^{2} \partial_{a} \psi \sigma^{a} \bar{\theta}-\frac{\mathrm{i}}{2} \bar{\theta}^{2} \theta \sigma^{a} \partial_{a} \bar{\psi}-\frac{1}{4} \theta^{2} \bar{\theta}^{2} \square \varphi
$$

where $H^{a}$ is the Hodge-dual of the field strength for a gauge two-form,

$$
\partial_{a} H^{a}=0
$$

From the superfield action

$$
S=-\int \mathrm{d}^{4} x \mathrm{~d}^{2} \theta \mathrm{d}^{2} \bar{\theta} G^{2}
$$

we read off the component Lagrangian

$$
\mathcal{L}=-2|\mu|^{2}-\frac{1}{2} \partial^{a} \varphi \partial_{a} \varphi-\mathrm{i} \psi \sigma^{a} \partial_{a} \bar{\psi}+\frac{1}{2} H^{a} H_{a} .
$$

The constant term in $\mathcal{L}$ indicates that a positive cosmological constant is generated once the system is lifted to supergravity.

We also subject $\boldsymbol{G}$ to the cubic nilpotency condition

$$
G^{3}=0 .
$$

The top component of this constraint can be written in the form

$$
\left(a-\frac{1}{4} \varphi \square \varphi\right) \varphi=\frac{1}{2} b,
$$

where

$$
\begin{aligned}
a & =2|\mu|^{2}+\frac{\mathrm{i}}{2}\left(\psi \sigma^{a} \partial_{a} \bar{\psi}-\partial_{a} \psi \sigma^{a} \bar{\psi}\right)-\frac{1}{2} H^{a} H_{a} \\
b & =\mu \psi^{2}+\bar{\mu} \bar{\psi}^{2}-H^{a} \psi \sigma_{a} \bar{\psi}
\end{aligned}
$$

It holds that

$$
b^{2}=a \psi^{2} \bar{\psi}^{2}, \quad b^{3}=0 .
$$


Equation (3.7a) is analogous to the one derived in [35] for the case of $\mathcal{N}=2 \rightarrow \mathcal{N}=1$ spontaneous supersymmetry breaking. Therefore, eq. (3.7a) may be solved similarly to the approach employed in [35]. Specifically, we have to look for a solution of the form

$$
\varphi=\mathcal{U} \psi^{2}+\overline{\mathcal{U}} \bar{\psi}^{2}+\mathcal{V}^{a} \psi \sigma_{a} \bar{\psi},
$$

with $\mathcal{U}$ and $\mathcal{V}^{a}$ being some composites of the dynamical fields $\psi_{\alpha}, \bar{\psi}_{\dot{\alpha}}$ and $H^{a}$. The point is that it is the ansatz (3.9) which is consistent with the three lowest components of (3.6), which are $\varphi^{3}=0, \varphi^{2} \psi_{\alpha}=0$ and $\varphi^{2} \bar{\psi}_{\dot{\alpha}}=0$. Now it follows from (3.7a) that

$$
\varphi^{2}=\frac{\psi^{2} \bar{\psi}^{2}}{4 a}=\left(\frac{b}{2 a}\right)^{2}
$$

This relation implies that the general solution of $(3.7 \mathrm{a})$ is

$$
\varphi=\frac{b}{2 a}+\frac{b^{2}}{32 a^{4}} \square b .
$$

The solution is well defined provided

$$
a_{0}:=2|\mu|^{2}-\frac{1}{2} H^{a} H_{a} \neq 0 .
$$

Making use of (3.11), the Lagrangian (3.5) turns into

$$
\mathcal{L}=-2|\mu|^{2}+\frac{b}{8 a} \square \frac{b}{a}+\frac{b^{2}}{64 a^{5}}(\square b)^{2}-\mathrm{i} \psi \sigma^{a} \partial_{a} \bar{\psi}+\frac{1}{2} H^{a} H_{a},
$$

modulo a total derivative. This Lagrangian depends on $H^{a}$ in a highly nonlinear way. However, all nonlinear contributions contain fermionic factors of $\psi_{\alpha}$ and $\bar{\psi}_{\dot{\alpha}}$. As a result, it is possible to dualise the gauge two-form, described by its gauge-invariant field strength $H^{a}$, into an axion $\mathcal{S}$ by considering the first-order model

$$
\mathcal{L}_{\text {first-order }}=\mathcal{L}(H)-H^{a} \partial_{a} \mathcal{S}
$$

in which $H^{a}$ is an unconstrained vector field, and $\mathcal{L}(H)$ stands for the Lagrangian (3.13).

\section{Nilpotent tensor multiplet coupled to a three-form multiplet}

The construction given in the pervious section admits a natural generalisation. The idea is that the complex parameter $\mu$ in (3.1) may be viewed as the expectation value of a chiral superfield. Therefore, a more general constraint is given by

$$
-\frac{1}{4} \bar{D}^{2} \boldsymbol{G}=\mathcal{Y}, \quad \overline{\mathcal{D}}_{\dot{\alpha}} \mathcal{Y}=0,
$$

for some background chiral superfield

$$
\mathcal{Y}(x, \theta, \bar{\theta})=\mathrm{e}^{\mathrm{i} \theta \sigma^{a} \bar{\theta} \partial_{a}}\left(\mu(x)+\theta^{\alpha} \rho_{\alpha}(x)+\theta^{2} F(x)\right) .
$$

Due to the identity

$$
-\frac{1}{4}\left[D^{2}, \bar{D}^{2}\right]=\mathrm{i} \partial_{\alpha \dot{\alpha}}\left[D^{\alpha}, \bar{D}^{\dot{\alpha}}\right]
$$


in order for $\boldsymbol{G}$ to contain a conserved vector field, $\mathcal{Y}$ has to be a three-form multiplet, which means that locally

$$
\mathcal{Y}=-\frac{1}{4} \bar{D}^{2} U, \quad \bar{U}=U,
$$

where $U$ is a real but otherwise unconstrained prepotential. The only implication of the representation (4.4) is that the auxiliary field $F$ in (4.2) is not an arbitrary complex field, but instead has the form

$$
F=d+\frac{\mathrm{i}}{2} \partial_{a} c^{a},
$$

where $d$ is a real scalar, and $c^{a}$ is the Hodge-dual of a gauge three-form. The general solution to the constraint (4.1) is

$$
\begin{aligned}
\boldsymbol{G}= & \varphi+\theta^{\alpha} \psi_{\alpha}+\bar{\theta}_{\dot{\alpha}} \bar{\psi}^{\dot{\alpha}}+\theta^{2} \bar{\mu}+\bar{\theta}^{2} \mu+\theta \sigma^{a} \bar{\theta}\left(H_{a}+c_{a}\right) \\
& +\theta^{2}\left(\bar{\rho}+\frac{\mathrm{i}}{2} \partial_{a} \psi \sigma^{a}\right) \bar{\theta}+\bar{\theta}^{2} \theta\left(\rho-\frac{\mathrm{i}}{2} \sigma^{a} \partial_{a} \bar{\psi}\right)+\theta^{2} \bar{\theta}^{2}\left(d-\frac{1}{4} \square \varphi\right),
\end{aligned}
$$

where $H_{a}$ obeys the constraint (3.3).

As in the previous section, we impose the cubic nilpotency condition

$$
G^{3}=0
$$

The top component of this constraint can be written in the form

$$
\left(\hat{a}+\varphi\left(d-\frac{1}{4} \square \varphi\right)\right) \varphi=\frac{1}{2} \hat{b},
$$

where

$$
\begin{aligned}
& \hat{a}=2|\mu|^{2}-\psi\left(\rho-\frac{\mathrm{i}}{2} \sigma^{a} \partial_{a} \bar{\psi}\right)-\left(\bar{\rho}+\frac{\mathrm{i}}{2} \partial_{a} \psi \sigma^{a}\right) \bar{\psi}-\frac{1}{2}(H+c)^{2}, \\
& \hat{b}=\mu \psi^{2}+\bar{\mu} \bar{\psi}^{2}-\left(H^{a}+c^{a}\right) \psi \sigma_{a} \bar{\psi} .
\end{aligned}
$$

As in the previous section, the lowest components of (4.7), $\varphi^{3}=0, \varphi^{2} \psi_{\alpha}=0$ and $\varphi^{2} \bar{\psi}_{\dot{\alpha}}=0$, imply that $\varphi$ has to have the form (3.9). Now it follows from (4.8a) that

$$
\varphi^{2}=\frac{\psi^{2} \bar{\psi}^{2}}{4 \hat{a}}=\left(\frac{\hat{b}}{2 \hat{a}}\right)^{2} .
$$

This relation implies that the general solution of $(3.7 \mathrm{a})$ is

$$
\varphi=\frac{\hat{b}}{2 \hat{a}}-\frac{\hat{b}^{2}}{4 \hat{a}^{3}} d+\frac{\hat{b}^{2}}{32 \hat{a}^{4}} \square \hat{b} .
$$

As an example of the construction given, we can choose $\mathcal{Y}$ of the form

$$
\mathcal{Y}=\mu+g \operatorname{tr}\left(W^{\alpha} W_{\alpha}\right),
$$

where $g$ is a real parameter, and $W_{\alpha}$ the covariantly chiral field strength of a Yang-Mills supermultiplet. Essentially, we are in a position to recycle the classic results on CherSimons couplings for linear multiplets, see, e.g., [41] for a review. 


\section{Coupling to three-form supergravity}

As is well known, every off-shell formulation for $\mathcal{N}=1$ supergravity can be realised as $\mathcal{N}=1$ conformal supergravity coupled to a compensating multiplet (see, e.g., [42, 43] for reviews). Different off-shell formulations correspond to choosing different compensators. As reviewed in [43], conformal supergravity can be described using the superspace geometry of $[44,45]$, which underlies the Wess-Zumino approach [46, 47] to old minimal supergravity $[48,49]$. This requires to extend the supergravity gauge group to include the super-Weyl transformations introduced in [50]. For the technical details, we refer the reader to the textbook [43], see also the recent paper [51]. The notation and conventions of [43] are used throughout this paper.

We start by reviewing the super-Weyl invariant formulation for three-form supergravity $[52,53]$, which was given in [54]. ${ }^{6}$ The corresponding conformal compensator is a three-form multiplet coupled to conformal supergravity. It is described by a covariantly chiral scalar $\Pi$ and its conjugate $\bar{\Pi}$, with $\Pi$ defined by

$$
\Pi=-\frac{1}{4}\left(\overline{\mathcal{D}}^{2}-4 R\right) P, \quad \bar{P}=P,
$$

where the scalar prepotential $P$ in (5.1) is real but otherwise unconstrained. ${ }^{7}$ The compensator $\Pi$ has to be nowhere vanishing so that $\Pi^{-1}$ exists. We postulate $P$ to be super-Weyl primary of weight $(1,1)$,

$$
\delta_{\sigma} P=(\sigma+\bar{\sigma}) P
$$

which implies that $\Pi$ is also primary,

$$
\delta_{\sigma} \Pi=3 \sigma \Pi \text {. }
$$

As follows (5.1), the prepotential $P$ is defined modulo gauge transformations of the form

$$
\delta_{L} P=L, \quad\left(\overline{\mathcal{D}}^{2}-4 R\right) L=0, \quad \bar{L}=L .
$$

The gauge parameter $L$ is a covariantly real linear superfield.

The action for three-form supergravity is

$$
\begin{aligned}
S_{\mathrm{SG}} & =-\frac{3}{\kappa^{2}} \int \mathrm{d}^{4} x \mathrm{~d}^{2} \theta \mathrm{d}^{2} \bar{\theta} E\left\{(\bar{\Pi} \Pi)^{\frac{1}{3}}-\frac{1}{2} m P\right\} \\
& =-\frac{3}{\kappa^{2}} \int \mathrm{d}^{4} x \mathrm{~d}^{2} \theta \mathrm{d}^{2} \bar{\theta} E(\bar{\Pi} \Pi)^{\frac{1}{3}}+\left\{\frac{m}{\kappa^{2}} \int \mathrm{d}^{4} x \mathrm{~d}^{2} \theta \mathcal{E} \Pi+\text { c.c. }\right\},
\end{aligned}
$$

where $m$ is a real parameter, and $E$ and $\mathcal{E}$ denote the full superspace and the chiral subspace integration densities, respectively. By construction the action is invariant under gauge transformations (5.3).

Complex three-form supergravity $[48,52,55]$ is obtained by choosing the prepotential $P$ in (5.1) to have the form

$$
P=\Gamma+\bar{\Gamma},
$$

\footnotetext{
${ }^{6}$ This formulation has been used in recent publications [14, 51, 55].

${ }^{7}$ The operator $\bar{\Delta}:=-\frac{1}{4}\left(\overline{\mathcal{D}}^{2}-4 R\right)$ is the covariantly chiral projection operator introduced in $[46,47]$.
} 
where $\Gamma$ is a covariantly complex linear scalar superfield constrained by

$$
\left(\overline{\mathcal{D}}^{2}-4 R\right) \Gamma=0 .
$$

Due to this constraint, the field strength (5.1) reads

$$
\Pi=-\frac{1}{4}\left(\overline{\mathcal{D}}^{2}-4 R\right) \bar{\Gamma} .
$$

The general solution to the constraint (5.6) is known [42] to be

$$
\Gamma=\overline{\mathcal{D}}_{\dot{\alpha}} \bar{\Psi}^{\dot{\alpha}}
$$

where $\bar{\Psi}^{\dot{\alpha}}$ is an unconstrained spinor superfield defined modulo gauge transformations

$$
\delta_{\Lambda} \bar{\Psi}^{\dot{\alpha}}=\overline{\mathcal{D}}_{\dot{\beta}} \bar{\Lambda}^{(\dot{\alpha} \dot{\beta})},
$$

which leave $\Gamma$ invariant. The super-Weyl transformation of $\bar{\Psi}^{\dot{\alpha}}$ is chosen to be [43]

$$
\delta_{\sigma} \bar{\Psi}^{\dot{\alpha}}=\frac{3}{2} \bar{\sigma} \bar{\Psi}^{\dot{\alpha}}
$$

and this transformation law implies

$$
\delta_{\sigma} \Gamma=(\sigma+\bar{\sigma}) \Gamma
$$

We define a deformed covariantly linear multiplet to obey the constraint

$$
-\frac{1}{4}\left(\overline{\mathcal{D}}^{2}-4 R\right) \boldsymbol{G}=f \Pi+\chi, \quad \overline{\mathcal{D}}_{\dot{\alpha}} \chi=0, \quad f=\text { const } .
$$

Here $\chi$ is a super-Weyl primary three-form multiplet, which means the following: (i) the super-Weyl transformation of $\chi$ is

$$
\delta_{\sigma} \chi=3 \sigma \chi
$$

and (ii) $\chi$ has the property

$$
\operatorname{Im} \int \mathrm{d}^{4} x \mathrm{~d}^{2} \theta \mathcal{E} \chi=0 .
$$

For instance, we can choose $\chi$ of the form

$$
\chi=g_{1} \operatorname{tr}\left(W^{\alpha} W_{\alpha}\right)+g_{2} W^{\alpha \beta \gamma} W_{\alpha \beta \gamma},
$$

where $g_{1}$ and $g_{2}$ are real parameters, and $W_{\alpha}$ the covariantly chiral field strength of a super Yang-Mills multiplet, and $W_{\alpha \beta \gamma}$ is the super-Weyl tensor [44, 45], see [43] for more details. The non-zero parameter $f$ in (5.12) is real (complex) provided the three-form multiplet $\Pi$ is real (complex). As in the rigid supersymmetric case, we subject $\boldsymbol{G}$ to the nilpotency condition

$$
G^{3}=0 .
$$

The action for the nilpotent tensor multiplet is

$$
S_{\mathrm{AG}}=-\int \mathrm{d}^{4} x \mathrm{~d}^{2} \theta \mathrm{d}^{2} \bar{\theta} E G^{2}(\bar{\Pi} \Pi)^{-\frac{1}{3}}
$$

The complete supergravity-matter action is $S=S_{\mathrm{SG}}+S_{\mathrm{AG}}+S_{\mathrm{SYM}}$, where $S_{\mathrm{SYM}}$ denotes the standard super Yang-Mills action in the presence of supergravity [46]. 


\section{Acknowledgments}

The author is grateful to Gabriele Tartaglino-Mazzucchelli for comments on the manuscript. Discussions with Dmitri Sorokin and Tsutomu Yanagida are gratefully acknowledged. The research presented in this work is supported in part by the Australian Research Council, project No. DP160103633.

\section{A More duality transformations}

In this appendix we discuss some generalisations of the duality transformations described in section 1. As an extension of the chiral model (1.3), we consider

$$
S=\int \mathrm{d}^{4} x \mathrm{~d}^{2} \theta \mathrm{d}^{2} \bar{\theta} \bar{\Phi} \Phi-\left\{\int \mathrm{d}^{4} x \mathrm{~d}^{2} \theta \Psi \Phi+\text { c.c. }\right\}, \quad \bar{D}_{\dot{\alpha}} \chi=0,
$$

where $\Psi$ is a background chiral superfield. This theory has a dual formulation that can be obtained by making use of the first-order action (1.4a), in which $\Sigma$ has to obey the constraint $^{8}$

$$
-\frac{1}{4} \bar{D}^{2} \Sigma=\Psi
$$

which is a deformation of (1.4b). The dual action has the form (1.5) with $\Sigma$ constrained according to (A.2). There exist dual formulations for chiral models that are obtained from (A.1) by replacing the superpotential by the rule $\Psi \Phi \rightarrow \Psi \Phi^{n}$, for an integer $n>2$. The dual actions are described in $[57,58]$ (such actions requires $\Phi$ to be nowhere vanishing).

A different duality transformation exists if the background chiral scalar $\Psi$ in (A.1) is a three-form multiplet,

$$
\Psi=-\frac{1}{4} \bar{D}^{2} U, \quad \bar{U}=U .
$$

Then the action (A.1) can be rewritten as an integral over the full superspace,

$$
S=\int \mathrm{d}^{4} x \mathrm{~d}^{2} \theta \mathrm{d}^{2} \bar{\theta}\{\bar{\Phi} \Phi-U(\Phi+\bar{\Phi})\}
$$

This action is obviously invariant under gauge transformations of the prepotential $U$ of the form

$$
\delta_{L} U=L, \quad \bar{D}^{2} L=0,
$$

with the chiral scalar $\Psi$ defined by (A.3) being a gauge-invariant field strength. Since the action (A.4) depends on $\Phi$ and $\bar{\Phi}$ only via the combination $\Phi+\bar{\Phi}$, the model naturally possesses a dual formulation given in terms of a real linear superfield $G$, see section 2 . The dual action is

$$
S=-\frac{1}{2} \int \mathrm{d}^{4} x \mathrm{~d}^{2} \theta \mathrm{d}^{2} \bar{\theta}(G+U)^{2} .
$$

\footnotetext{
${ }^{8}$ Constraints of the form (A.2) were introduced for the first time by Deo and Gates [56]. In the context of supergravity, such constraints were used in $[7,20]$ to generate couplings of the complex linear Goldstino superfield to chiral matter.
} 
It is invariant under the gauge transformation (A.5) provided $G$ transforms as

$$
\delta_{L} G=-L .
$$

The action (A.6) is constructed in terms of the gauge-invariant superfield $\mathfrak{G}:=G+U=\overline{\mathfrak{G}}$ obeying the constraints

$$
-\frac{1}{4} \bar{D}^{2} \mathfrak{G}=\Psi,
$$

which is a deformation of (1.7b). The same model can be naturally obtained by considering the first-order action (1.7a) in which $\mathfrak{G}$ is subject to the constraint (A.8).

Open Access. This article is distributed under the terms of the Creative Commons Attribution License (CC-BY 4.0), which permits any use, distribution and reproduction in any medium, provided the original author(s) and source are credited.

\section{References}

[1] I. Antoniadis, E. Dudas, S. Ferrara and A. Sagnotti, The Volkov-Akulov-Starobinsky supergravity, Phys. Lett. B 733 (2014) 32 [arXiv:1403.3269] [INSPIRE].

[2] S. Ferrara, R. Kallosh and A. Linde, Cosmology with nilpotent superfields, JHEP 10 (2014) 143 [arXiv: 1408.4096] [INSPIRE].

[3] G. Dall'Agata and F. Zwirner, On sgoldstino-less supergravity models of inflation, JHEP 12 (2014) 172 [arXiv:1411.2605] [INSPIRE].

[4] E. Dudas, S. Ferrara, A. Kehagias and A. Sagnotti, Properties of nilpotent supergravity, JHEP 09 (2015) 217 [arXiv: 1507.07842] [INSPIRE].

[5] E.A. Bergshoeff, D.Z. Freedman, R. Kallosh and A. Van Proeyen, Pure de Sitter supergravity, Phys. Rev. D 92 (2015) 085040 [arXiv:1507.08264] [INSPIRE].

[6] F. Hasegawa and Y. Yamada, Component action of nilpotent multiplet coupled to matter in 4 dimensional $\mathcal{N}=1$ supergravity, JHEP 10 (2015) 106 [arXiv:1507.08619] [INSPIRE].

[7] S.M. Kuzenko, Complex linear Goldstino superfield and supergravity, JHEP 10 (2015) 006 [arXiv: 1508.03190] [INSPIRE].

[8] R. Kallosh and T. Wrase, de Sitter supergravity model building, Phys. Rev. D 92 (2015) 105010 [arXiv: 1509.02137] [INSPIRE].

[9] M. Schillo, E. van der Woerd and T. Wrase, The general de Sitter supergravity component action, Fortsch. Phys. 64 (2016) 292 [arXiv:1511.01542] [InSPIRE].

[10] I. Bandos, L. Martucci, D. Sorokin and M. Tonin, Brane induced supersymmetry breaking and de Sitter supergravity, JHEP 02 (2016) 080 [arXiv: 1511.03024] [INSPIRE].

[11] F. Farakos, A. Kehagias, D. Racco and A. Riotto, Scanning of the supersymmetry breaking scale and the gravitino mass in supergravity, JHEP 06 (2016) 120 [arXiv:1605.07631] [INSPIRE].

[12] I. Bandos, M. Heller, S.M. Kuzenko, L. Martucci and D. Sorokin, The Goldstino brane, the constrained superfields and matter in $\mathcal{N}=1$ supergravity, JHEP 11 (2016) 109 [arXiv: 1608.05908] [INSPIRE]. 
[13] S.M. Kuzenko, I.N. McArthur and G. Tartaglino-Mazzucchelli, Goldstino superfields in $\mathcal{N}=2$ supergravity, JHEP 05 (2017) 061 [arXiv: 1702.02423] [INSPIRE].

[14] E.I. Buchbinder and S.M. Kuzenko, Three-form multiplet and supersymmetry breaking, JHEP 09 (2017) 089 [arXiv:1705.07700] [INSPIRE].

[15] U. Lindström and M. Roček, Constrained local superfields, Phys. Rev. D 19 (1979) 2300 [INSPIRE].

[16] M. Roček, Linearizing the Volkov-Akulov model, Phys. Rev. Lett. 41 (1978) 451 [INSPIRE].

[17] R. Casalbuoni, S. De Curtis, D. Dominici, F. Feruglio and R. Gatto, Nonlinear realization of supersymmetry algebra from supersymmetric constraint, Phys. Lett. B 220 (1989) 569 [INSPIRE].

[18] Z. Komargodski and N. Seiberg, From linear SUSY to constrained superfields, JHEP 09 (2009) 066 [arXiv:0907.2441] [INSPIRE].

[19] S. Samuel and J. Wess, A superfield formulation of the nonlinear realization of supersymmetry and its coupling to supergravity, Nucl. Phys. B 221 (1983) 153 [INSPIRE].

[20] S.M. Kuzenko and S.J. Tyler, Complex linear superfield as a model for Goldstino, JHEP 04 (2011) 057 [arXiv:1102.3042] [INSPIRE].

[21] D.V. Volkov and V.P. Akulov, Possible universal neutrino interaction, JETP Lett. 16 (1972) 438 [INSPIRE].

[22] D.V. Volkov and V.P. Akulov, Is the Neutrino a Goldstone Particle?, Phys. Lett. B 46 (1973) 109 [INSPIRE].

[23] V.P. Akulov and D.V. Volkov, Goldstone fields with spin 1/2, Theor. Math. Phys. 18 (1974) 28 [Teor. Mat. Fiz. 18 (1974) 39] [InSPIRE].

[24] P. Nath, Supersymmetry, supergravity, and unification, Cambridge University Press, (2017).

[25] E.A. Ivanov and A.A. Kapustnikov, General relationship between linear and nonlinear realizations of supersymmetry, J. Phys. A 11 (1978) 2375 [INSPIRE].

[26] S.M. Kuzenko and S.J. Tyler, On the Goldstino actions and their symmetries, JHEP 05 (2011) 055 and supplementary material [arXiv:1102.3043] [INSPIRE].

[27] S.M. Kuzenko and S.J. Tyler, Comments on the complex linear Goldstino superfield, arXiv: 1507.04593 [INSPIRE].

[28] F. Farakos, O. Hulík, P. Kočí and R. von Unge, Non-minimal scalar multiplets, supersymmetry breaking and dualities, JHEP 09 (2015) 177 [arXiv: 1507.01885] [INSPIRE].

[29] S. Ferrara, J. Wess and B. Zumino, Supergauge multiplets and superfields, Phys. Lett. B 51 (1974) 239 [INSPIRE].

[30] W. Siegel, Gauge spinor superfield as a scalar multiplet, Phys. Lett. B 85 (1979) 333 [INSPIRE].

[31] Y. Kahn, D.A. Roberts and J. Thaler, The goldstone and goldstino of supersymmetric inflation, JHEP 10 (2015) 001 [arXiv:1504.05958] [INSPIRE].

[32] S. Ferrara, R. Kallosh and J. Thaler, Cosmology with orthogonal nilpotent superfields, Phys. Rev. D 93 (2016) 043516 [arXiv: 1512.00545] [INSPIRE].

[33] J.J.M. Carrasco, R. Kallosh and A. Linde, Minimal supergravity inflation, Phys. Rev. D 93 (2016) 061301 [arXiv:1512.00546] [INSPIRE]. 
[34] G. Dall'Agata and F. Farakos, Constrained superfields in supergravity, JHEP 02 (2016) 101 [arXiv: 1512.02158] [INSPIRE].

[35] E. Dudas, S. Ferrara and A. Sagnotti, A superfield constraint for $\mathcal{N}=2 \rightarrow \mathcal{N}=0$ breaking, JHEP 08 (2017) 109 [arXiv: 1707.03414] [INSPIRE].

[36] S.M. Kuzenko and G. Tartaglino-Mazzucchelli, New nilpotent $\mathcal{N}=2$ superfields, Phys. Rev. D 97 (2018) 026003 [arXiv:1707.07390] [INSPIRE].

[37] U. Lindström and M. Roček, Scalar tensor duality and $N=1, N=2$ nonlinear $\sigma$-models, Nucl. Phys. B 222 (1983) 285 [inSPIRE].

[38] B. de Wit and M. Roček, Improved tensor multiplets, Phys. Lett. B 109 (1982) 439 [INSPIRE].

[39] S.J. Gates Jr., Super p-form gauge superfields, Nucl. Phys. B 184 (1981) 381 [INSPIRE].

[40] I.L. Buchbinder and S.M. Kuzenko, Quantization of the classically equivalent theories in the superspace of simple supergravity and quantum equivalence, Nucl. Phys. B 308 (1988) 162 [INSPIRE].

[41] P. Binetruy, G. Girardi and R. Grimm, Supergravity couplings: a geometric formulation, Phys. Rept. 343 (2001) 255 [hep-th/0005225] [INSPIRE].

[42] S.J. Gates Jr., M.T. Grisaru, M. Roček and W. Siegel, Superspace or one thousand and one lessons in supersymmetry, Front. Phys. 58 (1983) 1 [hep-th/0108200] [INSPIRE].

[43] I.L. Buchbinder and S.M. Kuzenko, Ideas and methods of supersymmetry and supergravity, or a walk through superspace, IOP, Bristol, (1995), (Revised Edition (1998)).

[44] R. Grimm, J. Wess and B. Zumino, Consistency checks on the superspace formulation of supergravity, Phys. Lett. B 73 (1978) 415 [INSPIRE].

[45] R. Grimm, J. Wess and B. Zumino, A complete solution of the Bianchi identities in superspace, Nucl. Phys. B 152 (1979) 255 [inSPIRE].

[46] J. Wess and B. Zumino, Superfield Lagrangian for supergravity, Phys. Lett. B 74 (1978) 51 [INSPIRE].

[47] B. Zumino, Supergravity and superspace, in Recent developments in gravitation - Cargèse 1978, M. Lévy and S. Deser eds., Plenum Press, N.Y., U.S.A., (1979), pp. 405-459.

[48] K.S. Stelle and P.C. West, Minimal auxiliary fields for supergravity, Phys. Lett. B 74 (1978) 330 [INSPIRE].

[49] S. Ferrara and P. van Nieuwenhuizen, The auxiliary fields of supergravity, Phys. Lett. B 74 (1978) 333 [INSPIRE].

[50] P.S. Howe and R.W. Tucker, Scale invariance in superspace, Phys. Lett. B 80 (1978) 138 [INSPIRE].

[51] S.M. Kuzenko and G. Tartaglino-Mazzucchelli, Complex three-form supergravity and membranes, JHEP 12 (2017) 005 [arXiv:1710.00535] [INSPIRE].

[52] S.J. Gates Jr. and W. Siegel, Variant superfield representations, Nucl. Phys. B 187 (1981) 389 [INSPIRE].

[53] B.A. Ovrut and D. Waldram, Membranes and three form supergravity, Nucl. Phys. B 506 (1997) 236 [hep-th/9704045] [INSPIRE]. 
[54] S.M. Kuzenko and S.A. McCarthy, On the component structure of $N=1$ supersymmetric nonlinear electrodynamics, JHEP 05 (2005) 012 [hep-th/0501172] [INSPIRE].

[55] F. Farakos, S. Lanza, L. Martucci and D. Sorokin, Three-forms in supergravity and flux compactifications, Eur. Phys. J. C 77 (2017) 602 [arXiv:1706.09422] [INSPIRE].

[56] B.B. Deo and S.J. Gates Jr., Comments on nonminimal $N=1$ scalar multiplets, Nucl. Phys. B 254 (1985) 187 [INSPIRE].

[57] D. Butter and S.M. Kuzenko, A dual formulation of supergravity-matter theories, Nucl. Phys. B 854 (2012) 1 [arXiv: 1106.3038] [INSPIRE].

[58] S.M. Kuzenko and G. Tartaglino-Mazzucchelli, Three-dimensional $N=2$ (AdS) supergravity and associated supercurrents, JHEP 12 (2011) 052 [arXiv:1109.0496] [INSPIRE]. 